\title{
DISTRIBUTION AND MIXING OF REAGENTS ON MULTICHANNEL PLASTIC CHIPS
}

\author{
Mingqi Zhao, Travis D. Boone, X. Charmaine Qiu, and Antonio J. Ricco* \\ ACLARA BioSciences, Inc. \\ 1288 Pear Avenue, Mountain View, CA 94043 \\ Fax: (650) 210-1210, email: aricco@aclara.com
}

\begin{abstract}
We report several methods to monitor and improve reagent distribution and mixing on an acrylic card designed to demonstrate multiplexed distribution. Results show that the even distribution of a reagent from a common central well to multiple channels is strongly dependent on the chemical state of the microchannel surfaces. We have characterized the performance in acrylic microchannels treated by solution, polymer adsorption, and chemical vapor-phase processes. We also describe scveral pumping systems built on our multiplex distribution cards that
\end{abstract}

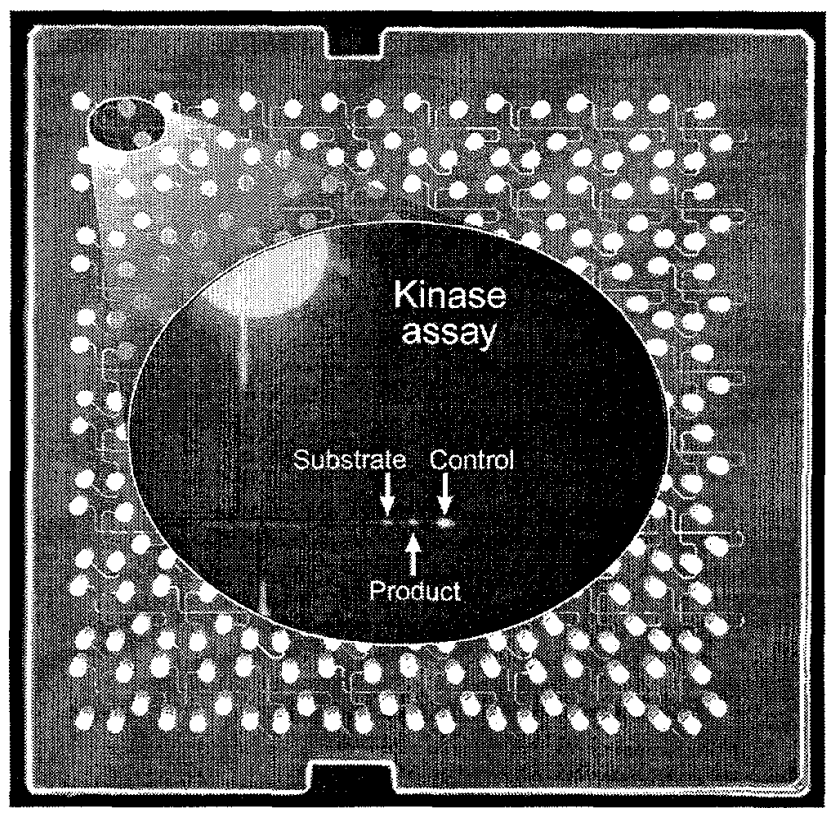

Figure 1. Fluorescent image of a 64-channel HTS assay card designed and produced at ACLARA. The key features of one channel are magnified in the center, showing sample injection and separation of the fluorescent marker ("Control"), the substrate, and the product formed by the action of a kinase enzyme on the substrate.

utilize electroosmotic flow as the motivating mechanism. By coating the appropriate channels with charged species, these systems have been demonstrated as a method of reagent distribution.

\section{INTRODUCTION}

Microfluidic devices integrating mixing, reaction, and separation functions have drawn great attention in the last decade because of their potential application to genetic analysis, pharmaceutical development, and medical diagnosis. Two critical technical problems are being addressed to hasten commercialization of these devices: (1) fabrication of plastic microchips by an economical method of mass-production; (2) reduction in the quantities of reagents used. Specifically, we have developed processes to fabricate microfluidic devices using inexpensive plastics and economical molding techniques. In most cases, the microfluidic chips require sub-microliter sample and reagent volumes, even for hour-long incubation times [1-3].

Disposable plastic microchips offer additional advantages for microanalytical systems [1-4]. For example, they minimize carryover and resultant cross-contamination, which is important for high-throughput screening (HTS) of pharmaceutical candidate compounds. In HTS, the action of an enzyme upon a substrate is quantified in the presence of each member of a "library" of hundreds of thousands of chemical compounds, the goal being to discover which library compounds significantly retard the enzyme/substrate reaction. On a single 96-channel disposable LabCard ${ }^{\text {TM }}$ chip developed at ACLARA, we can accomplish up to 96 separate assays in highly parallel fashion without crosscontamination.

If each assay is conducted in an independent fluidic "microcircuit," as shown in Figure 1, all reagents (cnzyme, substrate, test compound) must be dispensed individually into fluid reservoirs, requiring on the order of $100 \mathrm{~nL}$ of each reagent. To in particular-reagent distribution from a common reservoir shows promise. This strategy requires uniform distribution of reagents

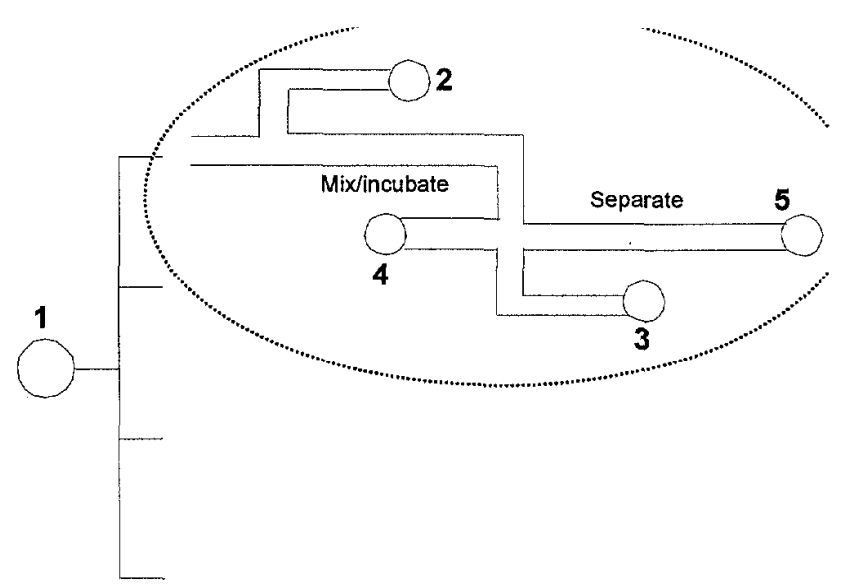

Figure 2. Schematic of the repetitive unit of a multichannel chip Reagent distribution, mixing, mixture incubation, sample injection and separation are integrated on one chip.

from the common reservoir to each of several channels, as well as efficient mixing of the enzyme and substrates on the distribution chip. The first part of this report describes several methods of distributing reagents evenly in microfluidic systems, and the 
second part describes methods to monitor and control the mixing of the reagents on the chip.

\section{EXPERIMENTAL DETAILS}

\section{Chip Design and Fabrication}

The multichannel chip used in this report has the repetitive unit shown in Figure 2. Reservoirs 1 and 2 contain enzyme and substrate, respectively, and buffers are added to reservoirs 3,4 , and 5 prior to analysis.

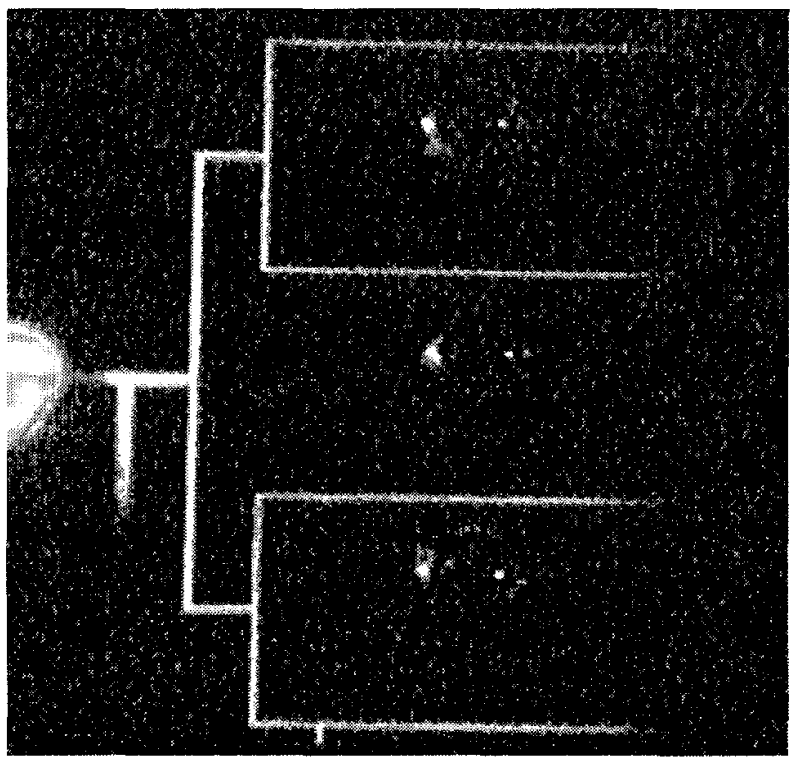

Figure 3. Electrokinetic distribution of fluorescein from a central well to 4 microchannels on a multichannel LabCard ${ }^{7 M}$ plate.

The card is fabricated by ACLARA by compression molding of plastic resin using a micromachined $\mathrm{Si}$ "master" wafer as the template and completed by lamination with a polymer film [3]. Distribution and mixing of rcagents are accomplished by electrokinetic motivation of the liquids [1-6]. Voltages applied to electrodes in the reservoirs are programmed for sample injection, mixing, and separation.

\section{Surface Modification of Microchannels}

Several surface treatment methods have been used to control the surface chemistry of the microchannels. Plasma treatment, chemical reaction, or adsorption of $\mathrm{OH}^{-}$is used to create fixed charge on acrylic surfaces. A surface bearing fixed charge of a given sign also can be used to bind charged polymers from solution to create an oppositely charged surface.

\section{RESULTS AND DISCUSSION}

\section{Distribution of Reagents}

The surface charge of microchannels in microfluidic chips determines the direction and velocity of electroosmotic flow (EOF) when a potential is applied; this effect is superimposed upon electrophoretically induced motion. Therefore, uniform distribution of reagent from a central well to multiple channels is strongly dependent on the chemical state of the microchannel surfaces. We have characterized the performance in acrylic microchannels that are untreated as well as those chemically treated by solution, polymer adsorption, and chemical vapor-phase plasma processes.

We use fluorescein (absorbance max.: $490 \mathrm{~nm}$, emission

\section{a: distribution of fluorescein to four channels}

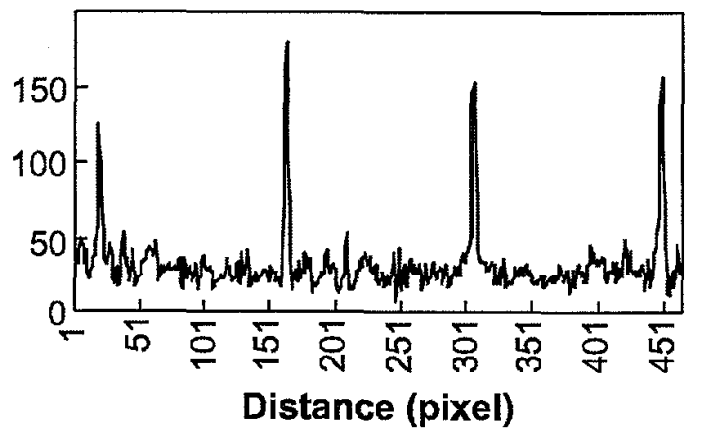

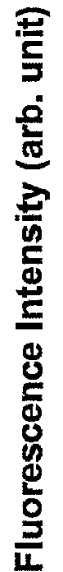

\section{b: stability of flow in reaction channel}

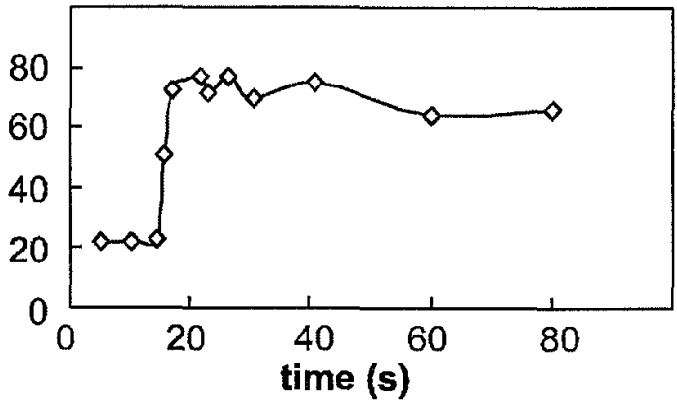

c: flow in separation channel

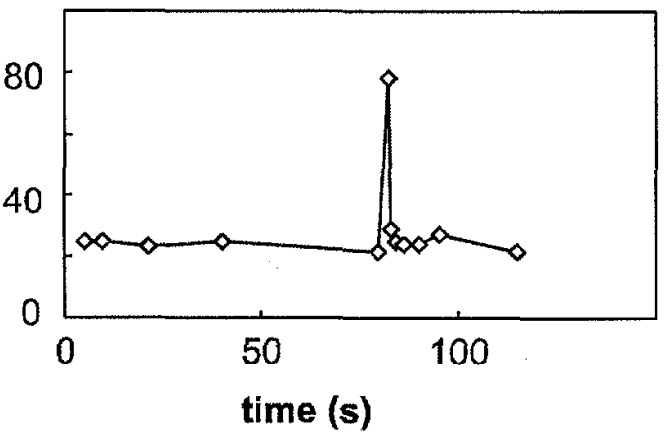

Figure 4. Digital data transferred from images recorded on $C C D$ camera, showing the distribution and separation using a 4-channel LabCard ${ }^{T M}$ device as shown in Figure 2: (a) flux of distributed 1 $m M$ fluorescein in the 4 reaction channels at steady state; (b) time course of fluorescence signal in one channel as $0.5 \mathrm{mM}$ fluorescein is distributed to it and several identical channels; (c) $0.5 \mathrm{mM}$ fluorescein plug in a separation channel following electrokinetic injection.

max.: $514 \mathrm{~nm}$ ) as a marker to reveal the distribution of reagent within the channels. The fluorescent images are obtained using a home-built imaging system. Although distribution of fluorescein 
is not perfectly uniform with untreated acrylic channels, distribution uniformity is improved significantly using aqueous $\mathrm{OH}^{-}$pretreatment, and the most stable, uniform distribution is achieved when the channel is coated with a charged polymer (Figure 3). Even distribution results are also observed for chips laminatcd with films pretreated with a gas-plasma discharge and having channels coated with positively charged polymers. If the channels are further modified with negatively charged polymers atop the positively charged polymers, the dye does not distribute as evenly from the central well 1 to the channels.

$$
\text { We developed a method to obtain quantitative }
$$

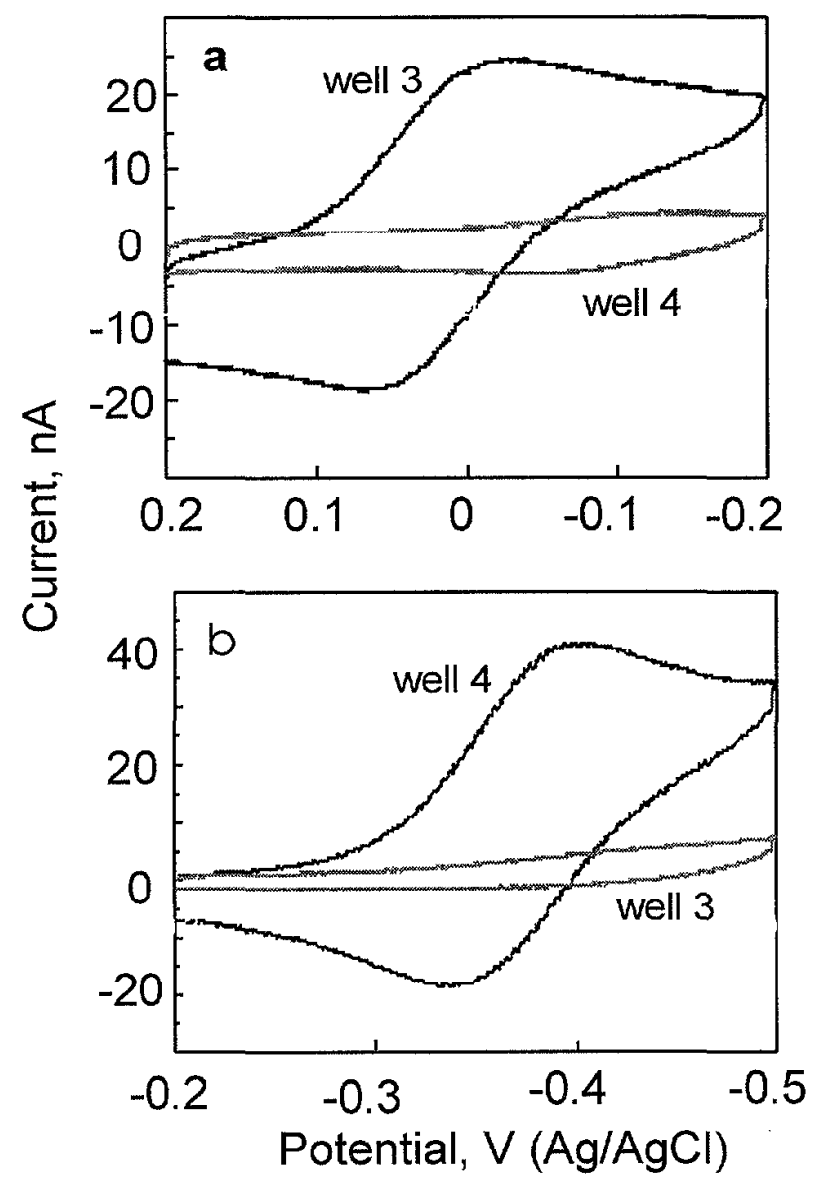

Figure 5. Electrochemical data showing that electroosmotic transport in the multiplexed distribution chip delivers reagents from the central well through the microchannels and to specific wells. The cyclic voltammetry of $\mathrm{Fe}(\mathrm{CN})_{6}^{3-}$ (a) and $\mathrm{Ru}\left(\mathrm{NH}_{3}\right)_{6}{ }^{3+}$ (b) are measured in wells 3 and 4, $70 \mathrm{~s}$ after initiating reagent distribution. $5 \mathrm{mM} \mathrm{Fe}(\mathrm{CN})_{6}^{3-}$ and $5 \mathrm{mM}$ $\mathrm{Ru}\left(\mathrm{NH}_{3}\right)_{6}{ }^{3+}$ are added to the central well in the case of $(a)$ and (b) respectively. A three-electrode bundle is placed in the wells 3 or 4 , which are $1.5 \mathrm{~mm}$ in diameter. The working and counter electrodes are $A u$ and $P t$ wires $0.25 \mathrm{~mm}$ in diameter. The reference electrode is $\mathrm{Ag} / \mathrm{AgCl}$ made from $0.25 \mathrm{~mm}$ diameter $\mathrm{Ag}$ wire.

information about reagent distribution on multichannel chips. In this method, images are recorded using a CCD camera and are then converted to digital format using XCAP (EPIX, Buffalo Grove, IL) and Image-Pro Plus software (Media Cybernetics, Silver Spring, MD). Figure 4a shows the flux of fluorescent molecules in each channel obtained by converting fluorescence intensity to a numerical value along a line perpendicular to the direction of flow through the four channcls on the multiplcxcd distribution card.

The results shown in Figure $4 \mathrm{a}$ indicate that the amount of fluorescein in each channel varies by no more than $20 \%$. By monitoring fluorescence intensity at a fixed position as a function of time, we have found the flow in the reaction channels to be quite stable (Figure 4b). Electrokinetically driven injection of the fluorescein from the reaction channel produces the expected

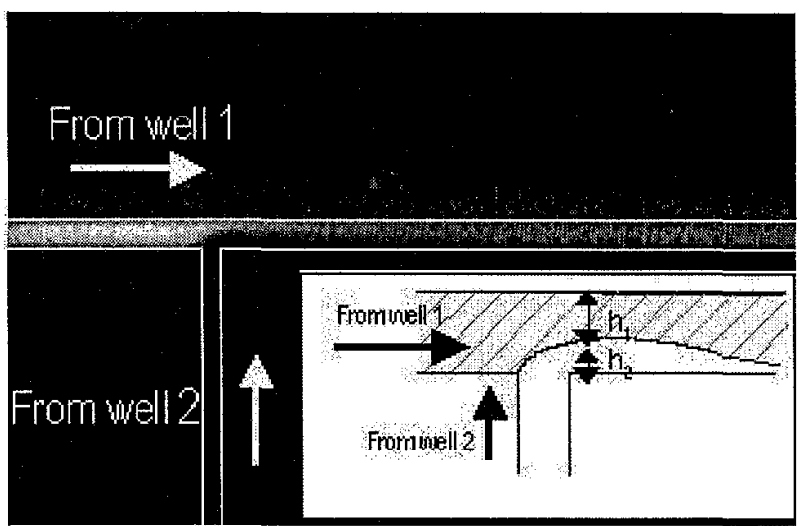

Figure 6. Mixing of reagents at a T-junction from two channels in a plastic fluidic card. The ratio of the mixing of reagents, $h 1 / h 2$, is almost equal to the ratio of the currents passing through the channels, Ia/Ib.

discrete fluorescent plug in the separation channel (Figure 4c).

Electrochemical measurements provide a method to monitor the distribution of electroactive species through microchannels to specific reservoirs, as shown by the results in Figure 5 . If $5 \mathrm{mM}$ $\mathrm{K}_{3} \mathrm{Fe}(\mathrm{CN})_{6}$ solution is added to the central well 1 and electroosmotically transported toward well 3 for $70 \mathrm{~s}$, a Faradaic current arising from of $\mathrm{Fe}(\mathrm{CN})_{6}{ }^{3-}$ reduction is subsequently observed in well 3 when it is probed electrochemically, no similar current being observed in well 4. Similarly, Figure 5 b shows the electrochemical detection of $\mathrm{Ru}\left(\mathrm{NH}_{3}\right)_{6} \mathrm{Cl}_{3}$ that was electroosmotically transported from well 1 to well 4 . The current was measured in both cases using a cluster of three electrodes bound together in such a way that the cross section was about 1 $\mathrm{mm}$.

\section{Mixing of Reagents}

For chips configured as shown in Figure 2, the reagent from the central well must be mixed and reacted with reagents from the side wells in the reaction channel prior to separation. 'T'o efficiently use expensive reagents such as enzymes, it is important to tightly control the ratio of the two reagents in the reaction channel. The ratio of mixing of reagents from two wells can be estimated by measuring the ratio of the widths of the two fluids in the channel at the mixing point using data from the CCD camera (Figure 6). As shown in Table 1, the ratio of the heights $\left(h_{1} / h_{2}\right)$ of the intensity peaks for the two channels agrees with the ratio of the current passing through the channels. Therefore, by using the current as a measurement parameter, it is possible to control the 
ratio of mixing of two reagents by adjusting the voltages applied to the driving electrodes.

Table 1. Relationship between applied voltages applied to three reservoirs, ratio of currents in the two corresponding channels, and the height ratio of the fluid in the channel measured from the CCD images. *

\begin{tabular}{ccc}
\hline $\mathrm{V}_{1}, \mathrm{~V}_{2}, \mathrm{~V}_{3}$ (Volts)** & $\mathrm{I}_{\mathrm{a}} / \mathrm{I}_{\mathrm{b}}^{* * *}$ & $\mathrm{~h}_{2} / \mathrm{h}_{1}$ \\
\hline $0,0,1000$ & 2.9 & $\sim 4.5$ \\
$50,0,1000$ & 1.9 & 1.7 \\
$100,0,1000$ & 1.2 & 0.9 \\
$150,0,1000$ & 0.75 & - \\
$200,0,1000$ & 0.42 & 0.46 \\
$250,0,1000$ & 0.16 & - \\
$300,0,1000$ & 0.05 & $\sim 0$ \\
\hline
\end{tabular}

*1 mM fluorescein solution is added to well 1 and buffer is added to well 2 . The channel surfaces are positively charged.

$* * V_{1}: V_{2}: V_{3}$ is the the voltages of the electrodes in wells 1,2 , and 3.

*** $I_{a}, I_{b}$ are the current from well 1 to 3 and from well 2 to $\mathbf{3}$, respectively.

\section{CONCLUSIONS}

We have shown that a plastic multiplexed distribution card can be fabricated and that uniform distribution of reagents can be achieved by tailoring the properties of the channel surfaces using polymers and/or the plasma treatment of the laminated films. Electroosmotic flow has been demonstrated on multichannel chips as a means to distribute and mix reagents without subjecting them directly to high electric fields. The mixing of reagents from two wells can be controlled by adjusting the current passing through the channels, which is achieved by tuning the voltages applied to the driving electrodes.

\section{REFERENCES}

1. R. M. McCormick, R. J. Nelson, M. G. Alonso-Amigo, D. J. Benvegnu, and H. H. Hooper, "Microchannel Electrophoretic Separations of DNA in Injection-Molded Plastic Substrates", Anal. Chem., 69, 2626 (1997).

2. C. S. Effenhauser, G. J. M. Bruin, and A. Paulus, "Integrated Chip-Based Capillary Electrophoresis", Electrophoresis, 18, 2203 (1997).

3. T. D. Boone, H. H. Hooper, and D. S. Soane, "Integrated Chemical Analysis on Plastic Microfluidic Devices", Technical Digest of the 1998 Solid-State Sensor and Actuator Workshop, Transducers Research Foundation, Cleveland (1998); pp. 87-92.

4. M. A. Roberts, J. S. Rossier, P. Bercier, and H. Girault, "UV Laser Machined Polymer Substrates for the Development of Microdiagnostic Systems" Anal. Chem., 69, 2035 (1997).

5. A. Manz, J. C. Fettinger, E. Verpoorte, H. Lüdi, H. M. Widmer, D. J. Harrison, "Micromachining of Monocrystalline Silicon and Glass for Chemical Analysis Systems - A Look into Next Century's Technology or just a Fashionable Craze?" Trends Anal. Chem., 10, 144 (1991).

6. D. J. Harrison, K. Fluri, K. Seiler, Z. Fan, C. S. Effenhauser, and A. Manz, "Micromachining a Miniaturized
Capillary Electrophoresis-Based Chemical Analysis System on a Chip", Science, 261, 895 (1993). 